EESTI NSV TEADUSTE AKADEEMIA TOIMETISED. 29. KOIDE FOUSIKA * MATEMAATIKA. 1980, NR. 3

ИЗВЕСТИЯ АКАДЕМИИ НАУК ЭСТОНСКОИ ССР. ТОМ 29 ФИЗИКА * МАТЕМАТИКА. 1980, № 3

A. ЭПШТЕИН

\title{
ОБОБЩЕННАЯ АВТОМОДЕЛЬНАЯ ЗАДАЧА О ТУРБУЛЕНТНОМ ВИХРЕВОМ КОЛЬЦЕ
}

\author{
(Представил Н. Эпик)
}

Движение и перенос тепла в турбулентном вихревом кольце, развивающемся в безграничной несжимаемой жидкости при условиях, когда справедливы аппроксимации теории свободной конвекции, могут быть описаны в декартовой или цилиндрической системе координат $x 0 y$ (ось $x$ совпадает с направлением основного движения) следующей системой уравнений

$$
\begin{gathered}
\partial \zeta / \partial t+u(\partial \zeta / \partial x)+v(\partial \zeta / \partial y)-j(v \zeta) / y^{j}= \\
=-\beta g(\partial T / \partial y)+v_{t}\left[\partial^{2} \zeta / \partial x^{2}+\partial^{2} \zeta / \partial y^{2}+j \partial(\zeta / y) / \partial y\right], \\
\partial^{2} \psi / \partial x^{2}+\partial^{2} \psi / \partial y^{2}-j(\partial \psi / \partial y) / y=-y^{j \zeta} \\
(\zeta=\partial v / \partial x-\partial u / \partial y), \\
u=(\partial \psi / \partial y) / y^{j}, \quad v=-(\partial \psi / \partial x) / y^{j}, \\
\partial T / \partial t+u(\partial T / \partial x)+v(\partial T / \partial y)= \\
=a_{t}\left[\partial^{2} T / \partial x^{2} f \partial^{2} T / \partial y^{2}+j(\partial T / \partial y) / y^{j}\right]
\end{gathered}
$$

с граничными условиями:

$$
\begin{array}{lll}
\zeta=\psi=\partial T / \partial y=0 & \text { при } & y=0, \\
\zeta=\psi=T \rightarrow 0 & \text { при } & x^{2}+y^{2} \rightarrow \infty .
\end{array}
$$

Здесь $u, v-$ компоненты скорости по направлениям $x, y$; $\zeta-$ вихрь; $\psi-$ функция тока; $T$ - избыточная относительно невозмущенной окружающей среды температура; $\beta$ - коэффициент объемного расширения; $g$ - ускорение силы тяжести; $v_{t}, a_{t}$ - турбулентные аналоги коэффициентов кинематической вязкости и температуропроводности, которые предполагаются здесь зависящими только от времени $t ; j=0$ для плоского и $j=1$ для осесимметричного течений. Перенос пассивной примеси описывается уравнением, идентичным (4), но с заменой температуры на концентрацию примеси.

Для автомодельного режима развития кольца уравнения (1)-(4) могут быть преобразованы путем исключения из них в явном виде независимой переменной $t$. Қак следует из теории размерностей, автомодельный режим может существовать лишь тогда, когда движение вихревого кольца определяется либо исключительно его начальным импульсом $I_{0}$ (влияние плавучести, характеризуемое членом $\beta g(\partial T / \partial y)$ 
в уравнении (1), в этом случае отсутствует или пренебрежимо мало), либо исключительно начальной плавучестью $Q_{0}$ (или начальным теплосодержанием $Q_{T 0}=Q_{0} / \beta g$; начальный импульс равен нулю). Вихревое кольцо первого типа будем называть динамическим, а второго типа конвективным (движение кольца определяется процессом свободной конвекции). Автомодельные преобразования уравнений (1) и (4), осуществляемые путем отнесения входящих в эти уравнения размерных параметров к некоторым характерным масштабам длины $L(t)$, скорости $V(t)=\varkappa(d L / d t)(\varkappa-$ произвольная константа), температуры $\Theta(t)$ или к соответствующей комбинации этих масштабов, приводят к уравнениям следующего вида (безразмерные параметры обозначены штрихом):

$$
\begin{gathered}
-A \zeta^{\prime}+\left(x u^{\prime}-x^{\prime}\right)\left(\partial \zeta^{\prime} / \partial x^{\prime}\right)+\left(x v^{\prime}-y^{\prime}\right)\left(\partial \zeta^{\prime} / \partial y^{\prime}\right)-j x\left(v^{\prime} \zeta^{\prime}\right) / y^{\prime j}= \\
=-B\left(\partial T / \partial y^{\prime}\right)+x v_{t}^{\prime}\left[\partial^{2} \zeta^{\prime} / \partial x^{\prime 2}+\partial^{2} \zeta^{\prime} / \partial y^{\prime 2}+j \partial\left(\zeta^{\prime} / y^{\prime}\right) / \partial y^{\prime}\right], \\
-C T^{\prime}+\left(x u^{\prime}-x^{\prime}\right)\left(\partial T^{\prime} / \partial x^{\prime}\right)+\left(x v^{\prime}-y^{\prime}\right)\left(\partial T^{\prime} / \partial y^{\prime}\right)= \\
=x a_{t}^{\prime}\left[\partial^{2} T^{\prime} / \partial x^{\prime 2}+\partial^{2} T^{\prime} / \partial y^{\prime 2}+j\left(\partial T^{\prime} / \partial y^{\prime}\right) / y^{\prime} j\right.
\end{gathered}
$$

где $A, B, C$ - некоторые коэффициенты. Уравнения (2), (3) и граничные условия (5) по форме не изменяются.

Следует отметить, что уравнение (6) описывает в сущности течение относительно расширяющегося вихревого кольца, так как $\left(x u^{\prime}-x^{\prime}\right)$ и $\left(x v^{\prime}-y^{\prime}\right)$ представляют собой компоненты вектора относительной скорости течения ( $x^{\prime}$ и $y^{\prime}$ есть составляющие вектора скорости расширения в данной точке).

В работах $\left[{ }^{1-6}\right]$, посвященных постановке и численному решению соответствующей задачи, в качестве условий нормировки, с помощью которых определялись масштабы $L, V, \Theta$, принимались условие сохранения импульса

$$
\left[(2 \pi)^{j} / 2\right] \int_{-\infty}^{\infty} \int_{0}^{\infty} \zeta y^{1+j} d x d y=I_{0}
$$

для динамического вихревого элемента (кольца или пары) и условие сохранения теплосодержания (или плавучести)

$$
(2 \pi) \int_{-\infty}^{j} \int_{0}^{\infty} T y^{j} d x d y=Q_{T 0}=Q_{0} / \beta g
$$

для конвективного вихревого элемента. Условие сохранения пассивной примеси идентично условию (9).

Законы сохранения (8), (9) подтверждены экспериментально $\left[{ }^{7-11}\right]$ для вихревых колец и пар с высоким начальным уровнем турбулентности, который в уравнениях $(6)$, (7) характеризуется коэффициентами $v_{t}^{\prime}$ и $a_{t}^{\prime}$. Однако из опытов с вихревыми кольцами «классического» типа, которые создаются без искусственной начальной турбулизации, явствует, что такие кольца движутся, испытывая как бы сопротивление окружающей среды $\left[{ }^{12}\right]$. Другие опыты указывают на значительную потерю такими кольцами примеси на всем пути их движения [ $\left.{ }^{13}\right]$. Численные расчеты для динамических вихревых пар $\left[{ }^{3-5}\right]$ также показали, что при $v_{t}^{\prime}$, ниже некоторого критического значения, образуется полузамкнутая нулевая поверхность тока $S(t)$, разделяющая относительное течение на две автономные зоны (см. рисунок). Из-за наличия на этой поверхности ненулевых градиентов вихря и темпіературы (концентрации 
примеси) происходит турбулентная диффузия завихренности и тепла (примеси) из внутренней зоны во внешнюю с образованием за вихревым кольцом турбулентного следа. В численных расчетах для конвективных вихревых элементов такого разделения течения получено не было, однако опытные данные $\left[{ }^{8,14}\right]$ указывают на возможность его существования и в данном случае. Будем называть внутреннюю зону - зоной собстственно вихревого кольца или просто зоной кольца, а внешнюю зону - зоной следа. При этом следует иметь в виду, что обе указанные зоны, помимо вихревого движения, включают в себя и потенциальные течения, индуцированные движением вихревого кольца.

Разделение относительного течения и, как следствие, потеря импульса и тепла (примеси) вихревым кольцом приводят к тому, что при обобщенной постановке задачи необходимо рассматривать зоны кольца и следа порознь, поскольку интегральные условия однозначности, а следовательно, и характерные масштабы движения будут для них различными.

Интегрируя уравнения (1) и (4) (умноженные, соответственно, на $y^{1+j}$ и

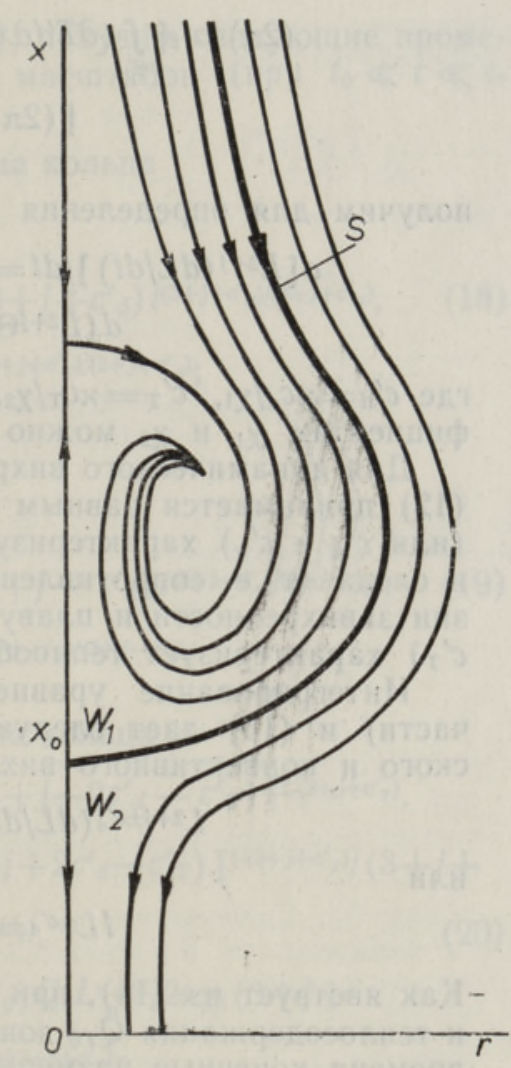
$\left.y^{j}\right)$ по зонам кольца $W_{1}(t)$ и следа $W_{2}(t)$ (интеграл берется от субстанциальной производной) с использованием уравнения неразрывности, получим

$$
\begin{gathered}
d I / d t=d\left\{\left[(2 \pi)^{j} / 2\right] \iint_{W_{1,2}(t)} \zeta y^{1+j} d x d y\right\} / d t=Q \mp F \mp M, \\
d Q_{T} / d t=d\left[(2 \pi)_{W_{t, 2}^{j}(t)}^{j} \int_{(t)} T y^{j} d x d y\right] / d t=\mp q,
\end{gathered}
$$

(здесь и далее верхний знак относится к зоне кольца, а нижний - к зоне следа), $Q=\beta g Q_{T} / 2(Q \approx 0$ для динамического вихревого элемента),

$$
F=\left[(2 \pi)^{j} v_{t} / 2\right]\left[\int_{S(t)}(\partial \zeta / \partial x) y^{1+j} d y+\int_{S(t)}(\partial \zeta / \partial y) y^{1+j} d x-\int_{S(t)} \zeta y^{j} d x\right]
$$

$M=\left[(2 \pi)^{j} \beta g / 2\right] \int_{S(t)} T y^{1+j} d x \quad(M=0$ для динамического вихревого элемента)

$$
q=(2 \pi)^{j} a_{t}\left[\int_{\mathbf{S}(t)}(\partial T / \partial x) y^{j} d y+\int_{\mathbf{S}(t)}(\partial T / \partial y) y^{j} d x\right]
$$

Переходя в (10) и (11) к безразмерным переменным и обозначая

$$
\left[(2 \pi)^{j} / 2\right] \iint_{W^{\prime}} \zeta^{\prime} y^{\prime 1+j} d x^{\prime} d y^{\prime}=\chi_{1}, \quad(2 \pi)^{j} \int_{W^{\prime}} T^{\prime} y^{\prime j} d x^{\prime} d y^{\prime}=\chi_{2},
$$

$\left[(2 \pi)^{j} v_{t}^{\prime} / 2\right]\left[\int_{S^{\prime}}\left(\partial \zeta^{\prime} / \partial x^{\prime}\right) y^{\prime 1+j} d y^{\prime}+\int_{S^{\prime}}\left(\partial \zeta^{\prime} / \partial y^{\prime}\right) y^{\prime 1+j} d x^{\prime}-\int_{S^{\prime}} \zeta^{\prime} y^{\prime j} d x^{\prime}\right]=c_{d}$, 


$$
\begin{gathered}
(2 \pi)^{j} a_{t}^{\prime}\left[\int_{S^{\prime}}\left(\partial T^{\prime} / \partial x^{\prime}\right) y^{\prime j} d y^{\prime}+\int_{S^{\prime}}\left(\partial T^{\prime} / \partial y^{\prime}\right) y^{\prime j} d x^{\prime}\right]=c_{T}, \\
{\left[(2 \pi)^{j / 2}\right] \int_{S^{\prime}} T^{\prime} y^{\prime 1+j} d x^{\prime}=c_{q},}
\end{gathered}
$$

получим для определения масштабов следующую систему уравнений:

$$
\begin{gathered}
d\left[L^{2+j}(d L / d t)\right] / d t=\beta g \chi_{2}^{\prime}\left(1 \mp c^{\prime}{ }_{q}\right) L^{2+j} \Theta \mp c_{d}^{\prime} L^{1+j}(d L / d t)^{2}, \\
d\left(L^{2+j} \Theta\right) / d t=\mp c^{\prime}{ }_{T} L^{1+j}(d L / d t) \Theta,
\end{gathered}
$$

где $c_{d}^{\prime}=x c_{d} / \chi_{1}, c_{T}^{\prime}=x_{T} / \chi_{2}, c^{\prime}{ }_{q}=c_{q} / \chi_{2}, \chi_{2}^{\prime}=\chi_{2} / \chi_{1}$. При этом коэффициентам $\chi_{1}$ и $\chi_{2}$ можно приписывать произвольные значения.

Для динамического вихревого элемента первый член в правой части (12) принимается равным нулю. Безразмерные коэффициенты $c_{d}$ и $c_{q}$ (или $c_{d}^{\prime}$ и $c_{q}^{\prime}$ ) характеризуют обмен импульсом между зонами кольца и следа (т. е. сопротивление движению кольца) в результате диффузии завихренности и плавучести соответственно. Коэффициент $c_{T}$ (или $c_{T}^{\prime}$ ) характеризует теплообмен между кольцом и следом.

Интеприрование уравнений (12) (без первого члена в правой части) и (13) дает следующие инварианты движения для динамического и конвективного вихревых элементов соответственно:

$$
L^{2+j \pm e^{\prime}}(d L / d t)=\text { const }, \quad L^{2+j \pm c^{\prime}}{ }_{T} \Theta=\text { const, }
$$

или

$$
I L \pm c_{d}^{\prime}=\text { const, } \quad Q_{T} L^{ \pm c_{T}^{\prime}}=\text { const. }
$$

Как явствует из (14), при конечных значениях начальных импульса $I_{0}$ и теплосодержания $Q_{T 0}$ зона 'кольца должна 'нметь 'в началыный момент времени конечные размеры, характеризуемые масштабом $L_{0}$ (который может быть сколь угодно малым, но отличным от нуля), а зона следа должна в пределе стремиться к конечным размерам, характеризуемым масштабом $L *$ (который может быть сколь угодно большим, но не бесконечным). Учитывая также, что в пределе все количество движения и тепло переходят из зоны кольца в след, можно (14) переписать в виде

$$
I L^{\mathrm{c}^{\prime}{ }_{a}}=I_{0} L_{0}^{\mathrm{c}^{\prime}{ }_{\alpha}}, \quad Q_{T} L^{\mathrm{c}^{\prime}}=Q_{T 0} L_{0}^{\mathrm{c}^{\prime}}
$$

для зоны кольца,

$$
I / L^{c^{\prime}} d=I_{0} / L_{*}^{c^{\prime} d}, \quad Q_{T} / L^{c^{\prime} r}=Q_{T 0} / L_{*}^{c_{*}^{\prime}}
$$

для зоны следа. Из (15) следует также

$$
L_{0} L_{*}=\text { const. }
$$

Таким образом, решения уравнений (12) и (13) должны удовлетворять следующим краевым условиям: а) зона кольца

$$
\begin{gathered}
\text { при } t=t_{0} \quad L=L_{0}, L^{2+j}(d L / d t)=I_{0} / \boldsymbol{x} \chi_{1} \quad \text { (динамический элемент) } \\
\text { или } L^{2+j}(d L / d t)=0 \text { (конвективный элемент), } \\
L^{2+j} \Theta=Q_{T 0} / \chi_{2} ;
\end{gathered}
$$

б) зона следа

при $t=t . \quad L=L \cdot, L^{2+j}(d L / d t)=I_{0} / x \chi_{1} \quad$ (динамический элемент),

$$
L^{2+j} \Theta=Q_{T 0} / \chi_{2} \text {. }
$$


Решая уравнения (12) и (13) при $(17 a, 6)$, получим следующие промежуточно-асимптотические зависимости для масштабов (при $t_{0} \ll t \ll t$. $\left.L_{0} \ll L \ll L_{*}\right)$.

1. Динамический вихревой элемент: а) зона кольца

$$
\begin{aligned}
& \left.L=\left[\left(3+j+c^{\prime}{ }_{d}\right) I_{0} L_{0}^{c^{\prime} a t / x \chi_{1}}\right]^{1 /\left(3+j+c^{\prime}\right.}{ }_{a}\right), \\
& V=x\left[\left(3+j+c^{\prime}{ }_{d}\right) I_{0} L_{a}^{c_{a}}{ }_{a} / \chi \chi_{1}\right]^{1 /\left(3+j+c^{\prime}{ }_{a}\right)} /\left(3+j+c^{\prime}{ }_{d}\right) t\left(2+j+c^{\prime}{ }_{a}\right) /\left(3+j+c^{\prime}{ }_{a}\right), \\
& \left.\Theta=Q_{T 0} L_{0}^{c^{\prime}{ }_{r}} / \chi_{2}\left[\left(3+j+c_{d}^{\prime}\right) I_{0} L_{0}^{c^{\prime}} a t / x \chi_{1}\right]^{\left(2+j+c^{\prime}{ }_{T}\right) /\left(3+j+c^{\prime}\right.}{ }_{a}\right) ;
\end{aligned}
$$

б) зона следа

$$
\begin{aligned}
& L=\left[\left(3+j-c_{d}^{\prime}\right) I_{0} t / \varkappa \chi_{1} L_{*}^{c^{\prime}}\right]^{1 /\left(3+j-c_{d}^{\prime}\right)}, \\
& \left.V=\chi\left[\left(3+j-c^{\prime}{ }_{d}\right) I_{0} / \chi \chi_{1} L_{{ }^{\prime}}^{c^{\prime}}\right]^{1 /\left(3+j-c_{d}^{\prime}\right.}\right) /\left(3+j-c_{d}^{\prime}\right) t\left(2+j-c_{d}^{\prime}\right) /\left(3+j-c_{d}^{\prime}\right) \text {, } \\
& \Theta=Q_{T 0} / \chi_{2} L_{*}^{c_{*}^{\prime}}\left[\left(3+j-c_{d}^{\prime}{ }_{d}\right) I_{0} t / x \chi_{1} L_{*}^{c^{\prime} d}\right]^{\left(2+j-c_{T}^{\prime}\right) /\left(3+j-c_{d}^{\prime}\right)} \text {. }
\end{aligned}
$$

2. Конвективный вихревой элемент: а) зона кольца

$$
L=\left[\left(3+j+c_{T}^{\prime}\right)^{2}\left(1-c^{\prime}{ }_{q}\right) Q_{0} L_{0}^{c^{\prime} t^{2}} / 2 \chi \chi_{1}\left(3+j+2 c^{\prime}{ }_{d}-c^{\prime}{ }_{T}\right)\right]^{1 /\left(3+j+c_{T}^{\prime}\right)},
$$$$
V=2 \varkappa\left[\left(3+j+c^{\prime}{ }_{T}\right)^{2}\left(1-c^{\prime}{ }_{q}\right) Q_{0} L_{c_{T}^{\prime}}^{c_{T}} / 2 \varkappa \chi_{1}\left(3+j+2 c^{\prime}{ }_{d}-c^{\prime}{ }_{T}\right)\right]^{1 /\left(3+j+c_{T}^{\prime}\right)} /(3+j+
$$

$$
\left.+c^{\prime}{ }_{T}\right) t\left(1+j+c^{\prime}\right) /\left(3+j+c^{\prime}{ }_{T}\right),
$$

$$
\Theta=Q_{0} L_{0}^{c^{\prime}}{ }_{T} / \chi_{2} \beta g \dot{g}\left[\left(3+j+c^{\prime}{ }_{T}\right)^{2}\left(1-c_{q}^{\prime}\right) Q_{0} L_{{ }_{0}}^{c_{x}} t^{2} / 2 \chi \chi_{1}(3+j+\right.
$$

$$
\left.\left.+2 c^{\prime}{ }_{d}-c_{T}^{\prime}\right)\right]^{\left(2+j+c_{T}^{\prime}\right) /\left(3+j+c_{r}^{\prime}\right)}
$$

б) зона следа

$$
\begin{aligned}
& L=\left[\left(3+j-c^{\prime}{ }_{T}\right)^{2}\left(1+c^{\prime}{ }_{q}\right) Q_{0} t^{2} / 2 \varkappa \chi_{1} L_{c_{T}}^{c_{T}}\left(3+j-2 c^{\prime}{ }_{d}+c^{\prime}{ }_{T}\right)\right]^{1 /\left(3+j-c^{\prime}{ }_{r}\right)}, \\
& V=2 \varkappa\left[\left(3+j-c^{\prime}\right)^{2}\left(1+c_{q}^{\prime}\right) Q_{0} / 2 \varkappa \chi_{1} L_{*}^{c_{T}^{\prime}}\left(3+j-2 c^{\prime}{ }_{d}+c^{\prime}{ }_{T}\right)\right]^{1 /\left(3+j-c^{\prime}{ }_{T}\right)} /(3+ \\
& \left.+j-c^{\prime} T\right) t\left(1+j-c_{T}^{\prime}\right)\left(3+j-c_{T}^{\prime}\right), \\
& \Theta=Q_{0} / \chi_{2} \beta g L_{*}^{c^{\prime}}\left[\left(3+j-c_{T}^{\prime}\right)^{2}\left(1+c^{\prime}{ }_{q}\right) Q_{0} t^{2} / 2 \chi_{\chi_{1}} L_{*}^{c^{\prime}{ }_{T}}(3+j-\right. \\
& \left.\left.-2 c^{\prime}{ }_{d}+c^{\prime}{ }_{T}\right)\right]^{\left(2+j-c_{T}^{\prime}\right) /\left(3+j-c^{\prime}{ }_{r}\right)} \text {. }
\end{aligned}
$$

С помощью выражений для $L$ в (18), (20) можно определить $t$. в зависимости от $L_{*}$, а затем с учетом $(15)$ - связь между $t$. и $t_{0}$.

В результате преобразования уравнений (1), (4) к виду (6), (7) с помощью (18)-(21) определяются численные значения коэффициентов $A, B, C$, которые приведены для различных случаев в таблице (при $\left.\chi_{1}=\chi_{2}=1\right)$.

Очевидно, что при отсутствии разделения течения, когда $c^{\prime}{ }_{d}=c^{\prime}{ }_{T}=$ $=c^{\prime}{ }_{q}=0, A, B, C$ приобретают значения, характерные для обычной постановки задачи $\left[{ }^{3-6}\right]$.

При обобщенной постановке задачи уравнения (6), (7) и (2), (3) должны решаться одновременно для зон кольца и следа, причем стыковка решений для отдельных зон осуществляется с помощью граничных условий, заданных на разделяющей поверхности тока $S^{\prime}$. Например,

$$
x^{\prime 2}+y^{\prime 2}=R^{\prime}\left(S^{\prime}\right) \quad \psi^{\prime}=\psi_{S}^{\prime}, \zeta^{\prime}=\zeta_{S}^{\prime}, T^{\prime}=T_{S}^{\prime} .
$$




\begin{tabular}{|c|c|c|c|c|c|}
\hline Вихревой & элемент & Зона & $A$ & $B$ & C \\
\hline \multirow{4}{*}{ Кольцо } & \multirow{2}{*}{$\begin{array}{l}\text { дичами- } \\
\text { ческое }\end{array}$} & кольца & $4+c_{d}^{\prime}$ & 0 & $3+c^{\prime} r$ \\
\hline & & следа & $4-c^{\prime}{ }_{d}$ & 0 & $3-c^{\prime} T$ \\
\hline & \multirow{2}{*}{$\begin{array}{l}\text { конвек- } \\
\text { тивное }\end{array}$} & кольца & $\left(4+c^{\prime} T\right) / 2$ & $\left(4+2 c_{d}^{\prime}-c_{T}^{\prime}\right) /\left(1-c_{q}^{\prime}\right)$ & $3+c^{\prime} T_{T}$ \\
\hline & & следа & $\left(4-c^{\prime} T\right) / 2$ & $\left(4-2 c^{\prime}{ }_{d}+c^{\prime} T\right) /\left(1+c_{q}^{\prime}\right)$ & $3-c^{\prime}{ }_{T}$ \\
\hline \multirow{4}{*}{ Пара } & \multirow{2}{*}{$\begin{array}{l}\text { динами- } \\
\text { ческая }\end{array}$} & кольца & $3+c_{d}^{\prime}$ & 0 & $2+c^{\prime}{ }_{T}$ \\
\hline & & следа & $3-c^{\prime}{ }_{d}$ & 0 & $2-c^{\prime} T$ \\
\hline & \multirow{2}{*}{$\begin{array}{l}\text { конвек- } \\
\text { тивная }\end{array}$} & кольца & $\left(3+c^{\prime} T_{T}\right) / 2$ & $\left(3+2 c^{\prime}{ }_{d}-c^{\prime}{ }_{T}\right) /\left(1-c^{\prime}{ }_{q}\right)$ & $2+c^{\prime}{ }_{T}$ \\
\hline & & следа & $\left(3-c^{\prime} x\right) / 2$ & $\left(3-2 c^{\prime}{ }_{d}+c^{\prime}{ }_{T}\right) /\left(1+c_{q}^{\prime}\right)$ & $2-c^{\prime}{ }_{T}$ \\
\hline
\end{tabular}

Для области кольца условия (22) являются внешними, а в качестве внутренних принимаются условия (5) на отрезке оси симметрии $(y=0)$ при $x^{\prime} \geqslant x_{0}^{\prime}$. Для области следа условия $(22)$ вместе с (5) на оси симметрии при $x^{\prime}<x_{0}^{\prime}$ являются внутренними, а в качестве внешних принимаются условия (5) на бесконечности. Кроме того, для определения $c_{d}^{\prime}, c^{\prime}{ }_{T}$ должны быть заданы и значения производных от $\zeta^{\prime}$ и $T^{\prime}$ на поверхности $S^{\prime}$. Рассмотренная постановка задачи является примером автомодельности второго рода [ $\left.{ }^{15}\right]$.

Принципиальная трудность решения обобщенной задачи заключается в том, что положение разделяющей поверхности $S^{\prime}$ в пространстве заранее неизвестно. Поэтому решение следует вести, вероятно, методом последовательных приближений. Предварительно задача решается без учета разделения относительного течения, и на основании полученного решения определяются в первом приближении координаты поверхности $S^{\prime}$, распределения параметров на этой поверхности и численные значения $c_{d}^{\prime}, c^{\prime}{ }_{T}$ и $c^{\prime}{ }_{q}$. С учетом этих данных решается уже задача в обобщенной постановке в следующем приближении и т. д.

\section{Л ИТЕРА Т У Р А}

1 Луговцов Б А., В сб.: Некоторые проблемы математики и механики, Л., «Наука», 1970 , с. $182-189$.

2. Lilly, D. K., J. Atmos. Sci., 21, № 1, 83-98 (1964).

3. К а лланский $Ф$. Б., Эпштейн А. М., Изв. АН СССР, Мех. жидкости и газа, № 1, 21-25 (1976).

4. К а пл ан ски й Ф. Б., Э пш т ей н А. М., В сб.: Процессы рассеивания вредных примесей в приземном слое атмосферы, Таллин, изд. АН ЭССР, 1976, c. $91-123$.

5. Epstein, A., Kaplanski, F., Rozpr. inz. - Eng. Trans., 28, № 1, 11-25 (1978).

6. Капланский Ф., Эпштейн А., Изв. АН ЭССР, Физ. Матем., 25, № 4, 408-417 (1976).

7. Richards, J. M., J. Fluid. Mech., 11, № 4, 369-384 (1961).

8. Rich a rds, J. M., Int. J. Air, Water, Poll., 7, № $1,17-34$ (1963).

9. Rich a rd s, J. M., J. Fluid. Mech., 21, № 1, 97-106 (1965).

10. T s a n g, G., Atmos. Environ., 5, № 3, 445-471 (1971).

11. W u, J., Phys. Fluids, 20, № 12, 1967-1974 (1977).

12. M a X w orthy, T., J. Fluid. Mech., 64, № 2, 227-239 (1974).

13. Т ар асов В. Ф., Якушев В. И., Прикл. мех. и техн. физ., № 1, 130-136 (1974). 
14. Wood w a rd, B., Quart. J. Roy. Meteorol. Soc., 85, № 2, 144-151 (1959).

15. Б а рен н л атт Г. И., Подобие, автомодельность, промежуточная асимптотика, Л., Гидрометеоиздат, 1978.

Институт термофизики и электрофизики Академии наук Эстонской ССР
Поступила в редакцию

$31 / \mathrm{I} \quad 1980$

\section{A. EPSTEIN}

\section{TURBULENTSE KEERISELISE RÕNGA OLDISTATUD ISEMODELLEERUV OLESANNE}

Artiklis on tuletatud liikumise invariantide, soojusülekande invariantide ja turbulentse keeriselise rõnga liikumisele iseloomulike mastaapide ajas muutumise seaduspärasused, arvestades rõnga liikumisimpulsi ja soojuse kadu rõnga järel tekkivas jäljes.

\section{A. EPSTEIN}

\section{A GENERALIZED PROBLEM OF THE SELF-SIMILAR TURBULENT VORTEX RING}

As experimental data indicate, the motion of non-buoyant and buoyant vortex rings usually occurs with a loss of momentum and heat (or passive admixture) to the wake at the rear of the vortex ring as a consequence of the separation of the relative velocity field. The theoretical models do not take this effect into account. However, the computations on the basis of these models clearly indicate the existence of a separating stream line if the turbulent viscosity is smaller than some critical value.

This paper deals with a generalization of existing theoretical models by means of adding the effect of separation. The invariants of motion and heat (admixture) transfer are established and the characteristic length, velocity, temperature scales for the vortex ring proper and the wake as functions of time are found by way of solving a set of differential equations based on the integral conditions of momentum and heat balance. The numerical values of coefficients in non-dimensionalized partial differential equations describing the motion and heat (admixfure) transfer in vortex rings, are calculated and the boundary conditions for the zones of ring and wake are formulated. 